\title{
Linear and Nonlinear Wave Propagation in Negative Refraction Meta-Materials
}

\author{
V. M. Agranovich ${ }^{1,2)}$, Y. R. Shen ${ }^{3)}$, R. H. Baughman ${ }^{1)}$, \\ A. A. Zakhidov ${ }^{1)}$ \\ 1 \\ UTD-NanoTech Institute, The University of Texas at Dallas, \\ Richardson TX, 75083-0688 USA \\ ${ }^{2}$ Institute of Spectroscopy, Russian Academy of Sciences, \\ Troitsk, Moscow obl. 142190, Russia \\ ${ }^{3}$ Physics Department, University of California, Berkeley, CA 94720 USA
}

\begin{abstract}
We discuss linear and nonlinear optical wave propagation in a left-handed medium (LHM) or medium of negative refraction (NRM). We use the approach of characterizing the medium response totally by a generalized electric polarization (with a dielectric permittivity $\tilde{\varepsilon}(\omega, \vec{k}))$ that can be decomposed into a curl and a non-curl part. The description has a one-to-one correspondence with the usual approach characterizing the LHM response with a dielectric permittivity $\varepsilon<0$ and a magnetic permeability $\mu<0$. The latter approach is less physically transparent in the optical frequency region because the usual definition of magnetization loses its physical meaning. Linear wave propagation in LHM or NRM is characterized by negative refraction and negative group velocity that could be clearly manifested by ultra-short pulse propagation in such a medium. Nonlinear optical effects in LHM can be predicted from the same calculations adopted for ordinary media using our general approach.
\end{abstract}

\section{Introduction.}

Over 30 years ago, Veselago [1] suggested that electromagnetic wave propagation in an isotropic medium with negative dielectric permittivity, $\varepsilon(\omega)<0$ and negative magnetic permeability $\mu(\omega)<0$ can exhibit very unusual properties. Since in such media, the wave vector $\vec{k}$, the electric field $\vec{E}$, and the magnetic field $\vec{H}$ of a wave form a left-handed orthogonal set, in contrast to the right- handed orthogonal set in an ordinary medium, they are sometimes labeled as left-handed meta-materials (LHM), as opposite to the ordinary right-handed media (RHM). Among the many interesting properties of wave propagation in such media are the appearances of a Pointing vector in the direction 
opposite to the wave vector $\vec{k}$ (or a negative group velocity) and a refracted wave on the same side of the surface normal as the incoming wave at an RHM/LHM interface (negative refraction) (so that the LHM is also called negative refraction medium (NRM)). The predictions of Veselago have aroused much theoretical interest and stimulated strong experimental efforts to create LHM or NRM in recent years [1-12]. Experimental success has been demonstrated in the microwave region [3, 6, 7]. More recently, it has been proposed that photonic-gap materials can behave as effective NRM at optical frequencies. Analogous to Bloch electron waves in the band structure of a crystal, optical waves in the periodic lattice of a photonic-gap material can have a Bloch state with its wave vector and group velocity in opposite directions [8-12]. Negative refraction of light at an air/photonic-crystal interface has been demonstrated in numerical simulations [1012].

Main emphasis of studies on LHM or NRM so far has been on linear optical effects. We consider here nonlinear optical processes in LHM and show that they also exhibit unusual properties with respect to energy conversion and propagation. We shall limit our discussion to homogeneous NRM excluding photonic-gap materials; the latter are more complex because of the presence of optical Umklapp processes.

Before discussing nonlinear optical effects in NRM we would like to note that there are usually two different approaches in dealing with wave propagation. One involves the set of fields $\vec{E}, \vec{D}, \vec{B}, \vec{H}$ with $\vec{D}=\varepsilon(\omega) \vec{E}$ and $\vec{B}=\mu(\omega) \vec{H}$ for monochromatic waves. This approach is often used in discussion of wave propagation in LHM or NRM, with the emphasis on the assumption that the response is completely characterized by $\varepsilon(\omega)<0$ and $\mu(\omega)<0$. However, it is known that while the approach is appropriate in the low frequency region, it is less so in the optical frequency region because $\mu(\omega)$ loses its usual physical meaning [13] and higher-order multipoles may become important. A more general approach is to use the set of fields $\vec{E}, \vec{D}, \vec{B}$ with $\vec{D}=\widetilde{\varepsilon} \vec{E}$ and $\vec{B}=\vec{H}$, satisfying the Maxwell equations:

$$
\nabla \times \vec{E}=\frac{i \omega}{c} \vec{B}, \nabla \times \vec{B}=-\frac{i \omega}{c} \vec{D}, \nabla \cdot \vec{D}=0, \nabla \cdot \vec{B}=0
$$

In this case, the linear optical response of the medium is completely characterized by the generalized dielectric constant $\widetilde{\varepsilon}(\omega, \vec{k})$, and the refractive index $n\left(\tilde{\varepsilon}=n^{2}\right)$ can always be taken as positive. We note that in this approach, the vectors $\vec{k}, \vec{E}$, and $\vec{B}$ always form a right-handed set, irrespective of the medium being NRM or not.

The only difference between wave propagation in bulk NRM and in ordinary media is the appearance of a negative group velocity in the former. As we shall see later, the negative group velocity is not limited to magnetic media with a negative magnetic permeability, but could exist in any dielectric media with a sufficiently strong and proper spatial dispersion. Thus the second approach is certainly more general and less confusing; in particular, it is better suited for description of nonlinear optical effects in NRM.

We show in Sec. II that there is a one-to-one correspondence between the E,D,H,B approach and the E,D,B approach. While the negative group velocity in LHM or NRM appears because $\varepsilon<0$ and $\mu<0$ in the former, it appears because $\tilde{\varepsilon}$ has a special and strong spatial dispersion in the latter. We then use the E,D,B approach to describe 
negative refraction at a RHM/LHM interface in Sec.III. The E, D, B approach is commonly used to formulate nonlinear optics in ordinary media. With this approach, the results can be easily converted to describe nonlinear optical effects in NRM. We discuss as examples, in Sec. IV and Sec. V, respectively, second harmonic generation (SHG) and stimulated Raman scattering in NRM. Finally, in Sec. VI, we consider briefly ultrashort pulse propagation in linear and nonlinear LHM (or NRM).

\section{Linear Wave Propagation in a Medium with a Generalized Response Coefficient.}

We present here the E, D, B approach generally used to describe optical wave propagation in a medium and make connection to the $\mathrm{E}, \mathrm{D}, \mathrm{H}, \mathrm{B}$ approach often used to describe wave propagation in NRM.

\section{A. Dielectric Tensor as the Response Coefficient.}

As pointed out by Landau and Lifshits [13], the magnetization $\vec{M}$ loses its usual physical meaning as magnetic moments per unit volume towards optical frequencies, and so does the magnetic permeability $\mu(\omega)$. It is then more appropriate to use the $\vec{E}, \vec{D}, \vec{B}$ approach with $\mu(\omega)$ set to be 1 . In this case, the linear response of a medium is fully described by the constitutive equation

$$
\vec{D}=\widetilde{\varepsilon}(\omega, \vec{k}) \vec{E}
$$

for a monochromatic wave, with $\widetilde{\varepsilon}$ being a generalized dielectric tensor that depends on both $\omega$ and $\vec{k}$.

To make connection with the E, D, B, H approach, we notice that in terms of multipole expansion, the displacement vector $\vec{D}$ takes the form [14]

$$
\frac{\partial}{\partial t} \vec{D}=\frac{\partial}{\partial t} \vec{E}+4 \pi\left[\frac{\partial}{\partial t} \vec{P}+c \nabla \times \vec{M}-\frac{\partial}{\partial t} \nabla \bullet \vec{Q}+\ldots\right]
$$

where $\vec{P}, \vec{M}$ and $\vec{Q}$ denote electric-dipole polarization, dipole magnetization, and electric quadrupole polarization, respectively. One can rewrite Eq. (3) into the form

$$
\frac{\partial}{\partial t} \vec{D}=\frac{\partial}{\partial t} \vec{E}+4 \pi\left[\frac{\partial}{\partial t} \vec{P}_{e f f}+c \nabla \times \vec{M}_{e f f}\right]
$$

with $\vec{P}_{\text {eff }}=\vec{P}-\nabla \bullet \vec{Q}+\ldots$ being a polar vector, and $\vec{M}_{\text {eff }}=\vec{M}-\nabla \square \vec{Q}_{M}+\ldots$ an axial vector. Here, we have purposely separated the field-induced response in the medium into the curl part and the non-curl part, each comprising all the associated multipoles. For linear response to monochromatic waves, we can then also introduce two response functions, $\vec{\varepsilon}_{\text {eff }}(\omega, \vec{k})$ and $\vec{\mu}_{\text {eff }}(\omega, \vec{k})$, defined by

$$
\begin{aligned}
& 4 \pi \vec{P}_{e f f}=\left[\vec{\varepsilon}_{e f f}(\omega, \vec{k})-1\right] \vec{E}, \\
& 4 \pi \vec{M}_{e f f}=\left[1-\vec{\mu}_{e f f}{ }^{-1}(\omega, \vec{k})\right] \vec{B} .
\end{aligned}
$$

This allows us to make connections to the E, D, H, B approach. If only the dipole terms are retained in the multipole expansion, we would have $\varepsilon_{\text {eff }}=\varepsilon(\omega)$ and $\mu_{\text {eff }}=\mu(\omega)$. With the help of $\nabla \times \vec{E}=-\frac{1}{c} \frac{\partial}{\partial t} \vec{B}$, we find, for a wave specified by $\omega$, and $\vec{k}$, 


$$
\widetilde{\varepsilon}(\omega, \vec{k}) \vec{E}=\vec{\varepsilon}_{\text {eff }}(\omega, \vec{k}) \vec{E}-\frac{c^{2}}{\omega^{2}} \vec{k} \times\left\{\left[1-\vec{\mu}_{e f f}^{-1}(\omega, \vec{k})\right](\vec{k} \times \vec{E})\right\}
$$

While the approach of using $\vec{D}, \vec{P}_{\text {eff }}$, and $\vec{M}_{\text {eff }}$ is more general, description in terms of multipoles may be useful in some cases, for example, in dealing with magneto-optical effects in magnetic crystals. The higher-order multipole terms, proportional to higher orders of $k$, are expected to be progressively much smaller than the lower-order terms at optical frequencies or lower.

To complete our discussion on the connection between the two approaches, we consider the special case of an isotropic medium with $\varepsilon_{\text {eff }}=\varepsilon$ and $\mu_{\text {eff }}=\mu$. Because of the spatial dispersion (dependence on $\vec{k}$ ) inherent in the magnetic dipole response, the generalized dielectric tensor $\tilde{\varepsilon}$ is anisotropic even though both $\varepsilon$ and $\mu$ are constant scalars. We find [13,15] from Eq. (6) that the longitudinal component of $\tilde{\varepsilon}$ along $\vec{k}$ and the transverse component of $\tilde{\varepsilon}$ perpendicular to $\vec{k}$ are

$$
\begin{gathered}
\tilde{\varepsilon}_{l}=\varepsilon \\
\tilde{\varepsilon}_{t r}=\varepsilon(\omega)+\frac{k^{2} c^{2}}{\omega^{2}}\left[1-\frac{1}{\mu(\omega)}\right] .
\end{gathered}
$$

For an electromagnetic wave with $\omega$ and $\vec{k}$ related by $k=\frac{\omega}{c} n$ and $n^{2}=\tilde{\varepsilon}_{t r}$, we obtain the known relation [13]

$$
n^{2}=\tilde{\varepsilon}_{t r}=\varepsilon(\omega) \mu(\omega)
$$

or more generally, one can show that

$$
\tilde{\varepsilon}_{t r}=\varepsilon_{e f f} \mu_{e f f}
$$

where $\varepsilon_{\text {eff }}$ and $\mu_{\text {eff }}$ refer to responses to the transverse wave. It is known that in general, in the optical frequency region, magnetic-dipole and electric-quadrupole responses should play equally important roles. This picture is not in the E, D, H, B approach for LHM presumably because the emphasis is on waves in the microwave region, but it is clear in the E, D, B approach with the generalized $\tilde{\varepsilon}$. Since the dependence of $\tilde{\varepsilon}(\omega, \vec{k})$ on $\vec{k}$ is often weak away from resonance even in the optical frequency region, we can expand $\tilde{\varepsilon}(\omega, \vec{k})$ into a power series,

$$
\widetilde{\varepsilon}_{i j}(\omega, \vec{k})=\varepsilon_{i j}(\omega)+\alpha_{i j l m}(\omega) k_{i} k_{j}+\cdots
$$

assuming a medium with inversion symmetry. The term quadratic in $k$ describes both the electric-quadrupole and the magnetic-dipole responses in the medium (although they have different symmetries in $\left.\tilde{\varepsilon}_{i j}(\omega, \vec{k})\right)$, and therefore has the two explicitly placed on equal footing.

\section{B. Poynting Vector, Energy Density, and Group Velocity}

In the $\vec{E}, \vec{D}, \vec{B}$ approach, the time-averaged electromagnetic energy density and Poynting vector in a medium (assuming isotropic for simplicity) are given by [13, 16] 


$$
\begin{aligned}
& \mathrm{U}=\frac{1}{16 \pi}\left[\frac{\partial(\omega \widetilde{\varepsilon})}{\partial \omega} \vec{E}^{*} \vec{E}+\vec{B}^{*} \vec{B}\right] \\
& \vec{S}=\frac{c}{16 \pi} \operatorname{Re}\left(\vec{E}^{*} \times \vec{B}\right)-\frac{\omega}{16 \pi} \nabla_{\vec{k}} \tilde{\varepsilon}(\omega, \vec{k}) \vec{E}^{*} \vec{E}
\end{aligned}
$$

which satisfy the energy conservation relation $\nabla \cdot \vec{S}+\frac{\partial U}{\partial t}=0$. Substitution of Eq. (7) into Eq. (11) leads to the same expression for the Poynting vector in the E,D,H,B approach with $\vec{B}=\mu \vec{H}$,

$$
\vec{S}=\frac{C}{8 \pi \mu} \operatorname{Re}\left(\vec{E}^{*} \times \vec{B}\right) .
$$

Knowing that in the latter case,

we find

$$
\nabla \cdot \vec{S}+\frac{1}{16 \pi} \frac{\partial}{\partial t}\left[\frac{\partial(\omega \varepsilon)}{\partial \omega} \vec{E}^{*} \vec{E}+\frac{\partial(\omega \mu)}{\partial \varpi} \vec{H}^{*} \vec{H}\right]=0
$$

$$
U=\frac{1}{16 \pi}\left[\frac{\partial(\omega \varepsilon)}{\partial \omega} \vec{E}^{*} \vec{E}+\frac{\partial(\omega \mu)}{\partial \varpi} \vec{H}^{*} \vec{H}\right] .
$$

as expected. More generally we can have $\varepsilon$ replaced by $\varepsilon_{\text {eff }}$ and $\mu$ by $\mu_{\text {eff }}$. Therefore, the two approaches are fully consistent in their descriptions of energy relations. This is true in general, irrespective of LHM or RHM.

However, Eqs. (10) and (11) are more general. In the E, D, H, B approach, the conditions for $\vec{S}$ to be opposite to $\vec{k}$ (or negative group velocity) in an LHM are $\varepsilon<0$ and $\mu<0$. In the E, D, B approach with the first term in Eq. (11) directed along $\vec{k}$, the negative group velocity requires the second term on the right-hand side of Eq. (11) dominate over the first term. This means that $\nabla_{\vec{k}} \tilde{\varepsilon}(\omega, \vec{k})$ must be sufficiently large and positive, or with $|\sqrt{\tilde{\varepsilon}} E|=|B|$ one must have $(\omega / c \sqrt{\tilde{\varepsilon}}) \nabla_{\vec{k}} \tilde{\varepsilon}(\omega, \vec{k})>1$. In addition, with $\tilde{\varepsilon}=\tilde{\varepsilon}^{\prime}+i \tilde{\varepsilon}^{\prime \prime}$, we must have $\tilde{\varepsilon}^{\prime \prime}$ negative for a lossy medium and positive for a gain medium. This is seen from the wave expression $\vec{E}=\vec{A} e^{i \vec{k} \cdot \vec{r}-i \omega t}$. For energy propagation in the direction opposite to $\vec{k}$, the imaginary part of $k=(\omega / c) \sqrt{\tilde{\varepsilon}}$, and hence $\tilde{\varepsilon}^{\prime \prime}$, must be negative for a wave attenuating along $-\vec{k}$, and positive for a wave growing along $-\vec{k}$. We can also see this explicitly from Eq. (8) in terms of $\varepsilon_{\text {eff }}$ and $\mu_{\text {eff }}$. For a lossy LHM medium, we have $\varepsilon_{\text {eff }}=\varepsilon_{\text {eff }}^{\prime}+i \varepsilon_{\text {eff }}^{\prime \prime}$ and $\mu_{\text {eff }}=\mu_{\text {eff }}^{\prime}+i \mu_{\text {eff }}^{\prime \prime}$ with $\varepsilon_{\text {eff }}^{\prime}<0, \varepsilon_{\text {eff }}^{\prime \prime}>0, \mu_{\text {eff }}^{\prime}<0$, and $\mu_{\text {eff }}^{\prime \prime}>0$. Knowing $\tilde{\varepsilon}(\omega, \vec{k})=\tilde{\varepsilon}^{\prime}+i \tilde{\varepsilon}^{\prime \prime}=\varepsilon_{\text {eff }} \mu_{\text {eff }}$, we find $\tilde{\varepsilon}^{\prime}=\varepsilon_{\text {eff }}^{\prime} \mu_{\text {eff }}^{\prime}-\varepsilon_{\text {eff }}^{\prime \prime} \mu_{\text {eff }}^{\prime \prime}>0$ for $\varepsilon_{\text {eff }}^{\prime \prime}<\left|\varepsilon_{\text {eff }}^{\prime}\right|$ and $\mu_{\text {eff }}^{\prime \prime}<\left|\mu_{\text {eff }}^{\prime}\right|$ and $\tilde{\varepsilon}^{\prime \prime}=\varepsilon_{\text {eff }}^{\prime \prime} \mu_{e f f}^{\prime}+\varepsilon_{\text {eff }}^{\prime} \mu_{e f f}^{\prime \prime}<0$.

To establish further connection between the two approaches, we notice that from Eq. (10), since the energy density must be positive, we must have $\frac{\partial[\omega \tilde{\varepsilon}(\omega, \vec{k})]}{\partial \omega}>0$ for given $\vec{k}$. Using Eq. (7), we find 


$$
\frac{\partial(\omega \varepsilon)}{\partial \omega}-\frac{k^{2} c^{2}}{\omega^{2}}\left(1-\frac{1}{\mu}\right)+\frac{k^{2} c^{2}}{\omega \mu^{2}} \frac{\partial(\omega \mu)}{\partial \omega}>0
$$

which reduces, with the help of $\frac{k^{2} c^{2}}{\omega^{2}}=n^{2}=\varepsilon \mu$, to

$$
\frac{\partial(\omega \varepsilon)}{\partial \omega}+\frac{\varepsilon}{\mu} \frac{\partial(\omega \mu)}{\partial \omega}>\varepsilon \mu
$$

It is seen that even if $\varepsilon<0$ and $\mu<0$ as in LHM, the quantity on the left hand side of Eq. (15) must still be positive. The above relation leads to a negative group velocity for wave propagation in an LHM: the group velocity is given by $\vec{v}_{g}=(\partial \omega / \partial k) \frac{\vec{k}}{k}$ with

$$
\frac{\partial k}{\partial \omega}=\frac{1}{2 k c^{2}} \frac{\partial\left(\omega^{2} \tilde{\varepsilon}\right)}{\partial \omega}=\frac{\omega \mu}{2 k c^{2}}\left[\frac{\partial(\omega \varepsilon)}{\partial \omega}+\frac{\varepsilon}{\mu} \frac{\partial(\omega \mu)}{\partial \omega}\right]
$$

which is negative since the quantity in the brackets is positive for $\varepsilon<0$ and $\mu<0$, the group velocity $\vec{v}_{g}$ is negative with respect to $\vec{k}$. So, here again, the two approaches are consistent.

\section{Transmission and Reflection at an RHM/LHM Interface}

While separation of optical response of a medium into $\varepsilon$ and $\mu$, and more generally, $\varepsilon_{\text {eff }}$ and $\mu_{\text {eff }}$ (from the non-curl part and the curl part of the response, respectively), may not be essential for wave propagation in the bulk, it is important for transmission and reflection of waves at an interface because of the boundary conditions on the fields. We consider here transmission and reflection of a wave with frequency $\omega$ at an air/LHM interface. The incoming wave is from the RHM side $(\mathrm{z}<0)$ with an incidence angle $\theta_{I}$, and the reflected and refracted waves have angles of reflection and refraction $\theta_{R}$ and $\theta_{T}$, respectively (Fig. 1; see also p.252 of Ref. [16]). We assume, for simplicity, the media are isotropic, all waves are s-polarized along $y$, and the longitudinal component of the waves can be neglected. 


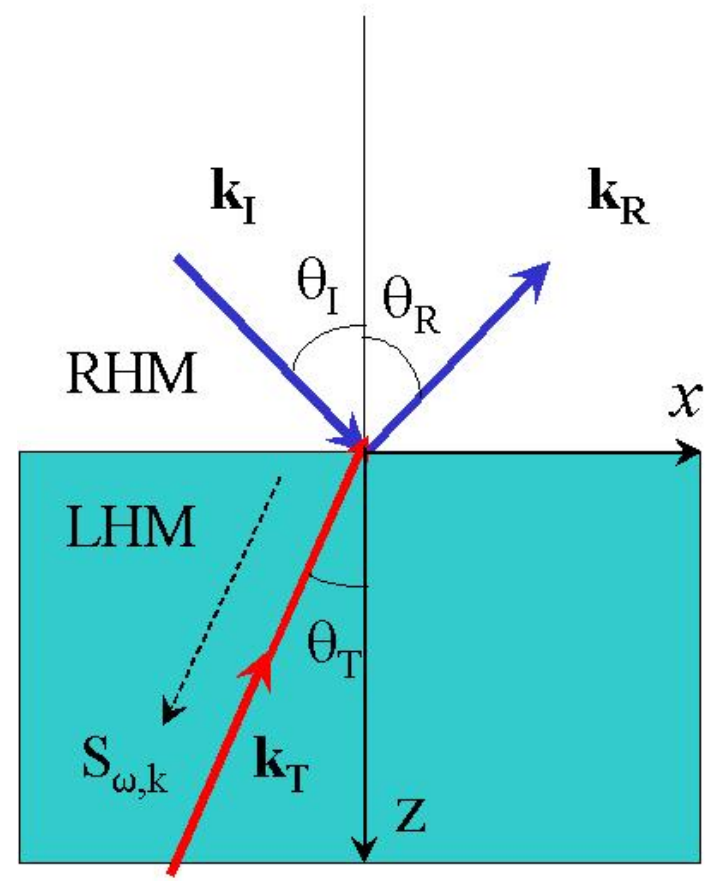

Fig.1. Geometry describing transmission and refraction of an incident wave at an RHM/LHM interface. Note that the Poynting vector $\vec{S}$ is in the opposite direction from $\vec{k}$ for the wave propagating in the LHM.

The $E$ field of the wave takes the form

$$
\begin{array}{rlrl}
\vec{E} & =\hat{y}\left[E_{I} e^{i \vec{k}_{R} \cdot \vec{r}}+E_{R} e^{i \vec{k}_{R} \cdot \vec{r}}\right] e^{-i \omega t}, & \mathrm{z}<0 & \\
& =\hat{y} E_{T} e^{i \vec{k}_{2} \cdot \vec{r}} e^{-i \omega t}, & \mathrm{z}>0
\end{array}
$$

with $\vec{k}_{I}=\left(\omega n_{1} / c\right)\left[\hat{x} \sin \theta_{I}+\hat{z} \cos \theta_{I}\right]$ and $\vec{k}_{R}=\left(\omega n_{1} / c\right)\left[\hat{x} \sin \theta_{I}-\hat{z} \cos \theta_{I}\right]$ in the RHM and $\vec{k}_{T}=\left(\omega n_{1} / c\right)\left[-\hat{x} \sin \theta_{\mathrm{T}}-\hat{z} \cos \theta_{T}\right]$ in the LHM. Both $n_{1}$ and $n_{2}$ are taken as positive. The boundary condition $k_{I x}=k_{T x}$ leads to the Snell's law for refraction,

$$
n_{1} \sin \theta_{I}=-n_{2} \sin \theta_{T}
$$

where the negative sign yields $\theta_{T}<0$. This means that the refracted wave appears on the same side of the surface normal as the incoming wave (i.e., negative refraction), as shown in Fig. 1. We have retained in Eq.(16) only the term that describes backward wave propagation in the LHM or NRM because as we mentioned earlier, the wave must decay away as $z \rightarrow \infty$ in the semi-infinite NRM medium. Given the E field in Eq.(16), the corresponding B field of the wave is obtained from $\vec{B}=($ ic $/ \omega) \nabla \times \vec{E}$.

The boundary conditions deduced from the Maxwell equations $\nabla \times \vec{E}=\frac{1}{c} \frac{\partial \vec{B}}{\partial t}$ and $\nabla \times\left(\vec{B}-4 \pi \vec{M}_{\text {eff }}\right)=\frac{1}{c} \frac{\partial}{\partial t}\left(\vec{E}+4 \pi \vec{P}_{\text {eff }}\right)$ are that $E_{y}$ and 
$B_{x}-4 \pi M_{\text {eff }, x}=B_{x} / \mu_{\text {eff }}$ must be continuous across the boundary. We then find from Eq.(16) the relations

$$
\begin{aligned}
& E_{I}+E_{R}=E_{T} \\
& n_{1}\left(E_{I}-E_{R}\right) \cos \theta_{I}=-\frac{n_{2}}{\mu_{\text {eff }}} E_{T} \cos \theta_{T}
\end{aligned}
$$

from which we obtain

$$
\begin{aligned}
& E_{R}=\frac{n_{1} \cos \theta_{I}+\left(n_{2} / \mu_{\text {eff }}\right) \cos \theta_{T}}{n_{1} \cos \theta_{I}-\left(n_{2} / \mu_{\text {eff }}\right) \cos \theta_{T}} E_{I} \\
& E_{T}=\frac{2 n_{1} \cos \theta_{I}}{n_{1} \cos \theta_{I}+\left(n_{2} / \mu_{\text {eff }}\right) \cos \theta_{T}} E_{I}
\end{aligned}
$$

Accordingly, the reflection and transmission coefficients are given by

$$
\begin{aligned}
& R=\frac{\left|E_{R}\right|^{2}}{\left|E_{I}\right|^{2}}=\left|\frac{n_{1} \cos \theta_{I}+\left(n_{2} / \mu_{\text {eff }}\right) \cos \theta_{T}}{n_{1} \cos \theta_{I}-\left(n_{2} / \mu_{\text {eff }}\right) \cos \theta_{T}}\right|^{2} \\
& T=-\frac{\left|E_{T}\right|^{2}\left(n_{2} / \mu_{\text {eff }}\right) \cos \theta_{T}}{\left|E_{I}\right|^{2} \cos \theta_{I}}=\frac{-4 n_{1} n_{2} / \mu_{\text {eff }} \cos \theta_{I} \cos \theta_{T}}{\left|n_{1} \cos \theta_{I}-\left(n_{2} / \mu_{\text {eff }}\right) \cos \theta_{T}\right|^{2}}
\end{aligned}
$$

As expected, we have $\mathrm{T}+\mathrm{R}=1$.

\section{Second Harmonic Generation from LHM}

We now consider a simple nonlinear optical effect: second harmonic generation (SHG) from a semi-infinite LHM medium with a non-vanishing nonlinear susceptibility $\ddot{\chi}^{(2)}$ in air $\left(n_{1}=1\right)$. We anticipate that the medium could be LHM at $\omega$ or $2 \omega$, but not at both $\omega$ and $2 \omega$, and consider here an LHM at $\omega$ only. The incoming fundamental wave at $\omega$ then has the same geometry as that depicted in Fig.1 with the air/medium boundary surface set at $\mathrm{z}=0$. It refracts negatively (as defined earlier) into the nonlinear medium and induces in the medium ( $\mathrm{z}>0$ ) a nonlinear polarization $\vec{P}^{(2)}(2 \omega)=\vec{\chi}^{(2)}: \vec{E}(\omega) \vec{E}(\omega)$, which is the source for SHG. For simplicity, we assume that the medium is isotropic and $\vec{P}^{(2)}(2 \omega)$ is parallel to the boundary surface along $y$ such that only the s-polarized field is generated at $2 \omega$. We also assume there is no input at $2 \omega$ and depletion of the pump field at $\omega$ is negligible. 


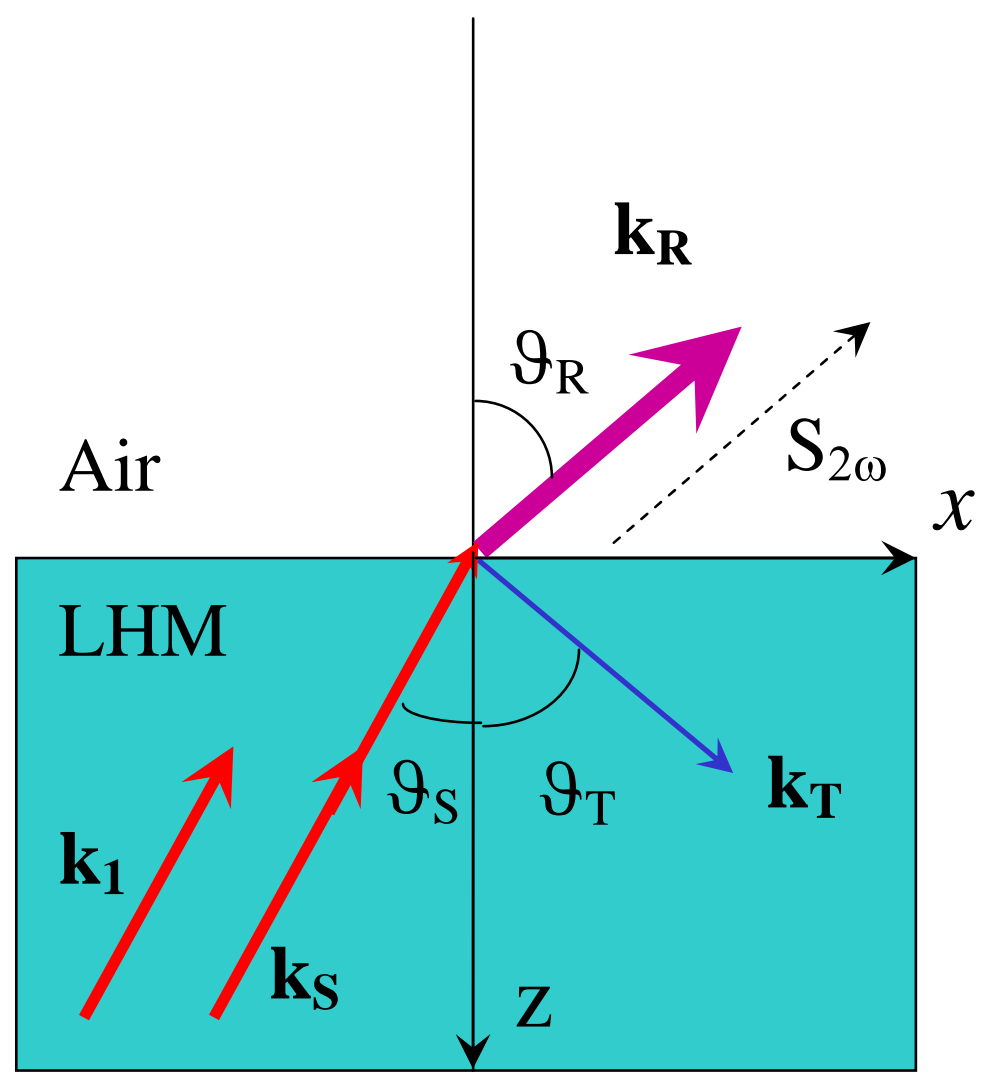

Fig.2. Second harmonic generation at an air/LHM interface. The wave vectors of the second harmonic waves are denoted by $\vec{k}_{S}, \vec{k}_{T}$, and $\vec{k}_{R}$, and the wave vector of the fundamental wave in LHM is $\vec{k}_{1}=\vec{k}_{S} / 2$.

Following the usual derivation of SHG using the E,D,B approach, we solve the wave equation for $\vec{E}(2 \omega)$ with $\vec{P}^{(2)}(2 \omega)$ as the source term and immediately find the following results [14]. Let the pump field in the LHM be described by $\vec{E}(\omega)=\vec{A}_{1} e^{i \vec{k}_{1} \cdot \vec{r}-i \omega t}$, with $k_{1 z}<0$ and $\vec{k}_{1}$ making an angle $\theta_{1 T}$ (as determined by the Snell's law of Eq.(17)) with the surface normal. The induced nonlinear polarization takes the form $\vec{P}^{(2)}(2 \omega)=\hat{y} \mathrm{P}^{(2)} e^{i \vec{k}_{s} \cdot \vec{r}-i 2 \omega t}$ with $\hat{y} \mathrm{P}^{(2)}=\vec{\chi}^{(2)}: \vec{A}_{1} \vec{A}_{1}$ and $\vec{k}_{S}=2 \vec{k}_{1}$. The transmitted SH wave $(\mathrm{z}>0)$ is then given by

$$
\begin{aligned}
\vec{E}_{T}(2 \omega) & =\hat{y}\left[A_{2 T} e^{i \vec{k}_{T} \cdot \vec{r}}+F e^{i \vec{k}_{S} \cdot \vec{r}}\right] e^{-i 2 \omega t} \\
A_{2 T} & =\frac{k_{R} \cos \vartheta_{R}+k_{S} \cos \vartheta_{S}}{k_{T} \cos \vartheta_{T}+k_{R} \cos \vartheta_{R}} F \\
F & =\frac{4 \pi(2 \omega)^{2} \mathrm{P}^{(2)}}{c^{2}\left(k_{S}^{2}-k_{T}^{2}\right)}
\end{aligned}
$$


where $\vec{k}_{T}$ and $\vec{k}_{R}$ are the wave vectors at $2 \omega$ of the transmitted homogeneous wave in the LHM and the reflected wave into the air side, respectively, with $k_{T}=2 \omega n_{2 \omega} / c$ and $k_{R}=2 \omega / c$. The angles $\vartheta_{T}$ and $\vartheta_{R}$ made by $\vec{k}_{T}$ and $\vec{k}_{R}$ with the surface normal (Fig.2,b) are obtained by matching of the wave vector components along the surface:

$$
k_{R} \sin \vartheta_{R}=k_{T} \sin \vartheta_{T}=k_{S} \sin \vartheta_{S}=2 k_{1} \sin \theta_{1 T}
$$

remembering that $k_{1 Z}<0$ and $k_{S Z}<0$. The reflected $\mathrm{SH}$ wave is given by

$$
\begin{aligned}
\vec{E}_{R}(2 \omega) & =\hat{y} A_{2 R} e^{i \vec{k}_{R} \cdot \vec{r}-i \omega t} \\
A_{2 R} & =\frac{k_{T Z}-k_{S Z}}{k_{T Z}+k_{R Z}} F
\end{aligned}
$$

The results given by Esq. (21)-(23) are the same as those for SHG in RHM [14] except that instead of $k_{1 Z}>0$ and $k_{S Z}>0$, we now have $k_{1 Z}<0$ and $k_{S Z}<0$. The physical consequence is that with $\vec{k}_{T}$ and $\vec{k}_{S}=2 \vec{k}_{1}$ nearly in opposite directions, the SHG process in the LHM is badly phase-mismatched and the SHG in transmission varies rapidly with $z$. It is then the SHG in reflection that is more interesting. As seen from Eqs.(21) and (23) $\}$, if $-k_{S Z}$ approaches $k_{T Z}$, the reflected SH output in an LHM can be much stronger than that from an RHM medium. The ratio of the Pointing vectors of reflected and transmitted SHG is

$$
\begin{aligned}
\frac{S_{R, 2 \omega}}{S_{T, 2 \omega}} & =\frac{1}{n_{2 \omega}}\left(\frac{A_{2 R}}{A_{2 T}}\right)^{2}=\frac{1}{n_{2 \omega}}\left(\frac{k_{T Z}-k_{S Z}}{k_{R Z}+k_{S Z}}\right)^{2} \\
& =\frac{1}{n_{2 \omega}}\left(\frac{n_{2 \omega} \cos \theta_{T}+n_{\omega} \cos \theta_{S}}{\cos \theta_{R}-n_{\omega} \cos \theta_{S}}\right)^{2}
\end{aligned}
$$

which can be very large if $\cos \vartheta_{R} \sim n_{\omega} \cos \vartheta_{S}$

If the medium is RHM at $\omega$ and LHM at $2 \omega$, the same results described in Eqs. (21) - (23) are still valid except that now $k_{S Z}>0$ and $k_{T Z}<0$, and $k_{T}$ should be replaced by $k_{T} / \mu_{\text {eff }}$. The reflected SH wave in the air appears on the same side of the surface normal as $\vec{k}_{T}$ (negative refraction), as dictated by the Snell's law of Eq.(17). Here again, the SHG in transmission is badly phase-mismatched, but for the reflected direction (relatively to the incident input fundamental wave propagation), SHG shows strong enhancement as $k_{R Z}$ approaches $-k_{T Z}$.

The above discussion can be easily generalized to other wave mixing processes and the results are the same if $\vec{P}^{(2)}(2 \omega)$ is replaced by $\vec{P}^{N L S}\left(\omega_{S}\right)$ as the induced nonlinear polarization at $\omega_{S}$ and the other quantities are changed accordingly.

\section{Stimulated Raman Scattering}

Stimulated Raman scattering in an LHM can also be easily described using the E, D, B approach. We consider the case where only the Stokes wave at frequency $\omega_{\mathrm{S}}$ sees the LHM. The stimulated Raman process pumped by $\vec{E}\left(\omega_{p}\right)$ in the semi-infinite medium 
covering $\mathrm{z} \geq 0$ is described by a third-order nonlinear susceptibility $\chi_{R}\left(\omega_{S}\right)=\chi_{R}^{\prime}-i \chi_{R}^{\prime \prime}$ with $\chi_{R}^{\prime \prime}>0$, which appears as a pump-intensity dependent term in $\varepsilon_{\text {eff }}$ with $\varepsilon_{\text {eff }}=\varepsilon_{\text {eff }}^{\prime}+i \varepsilon_{\text {eff }}^{\prime \prime}+4 \pi\left(\chi_{R}^{\prime}-i \chi_{R}^{\prime \prime}\right)\left|E\left(\omega_{p}\right)\right|^{2}$. Following Eq.(8), we have $\tilde{\varepsilon}=\tilde{\varepsilon}^{\prime}+i \tilde{\varepsilon}^{\prime \prime}$ with $\tilde{\varepsilon}^{\prime}=\varepsilon_{\text {eff }}^{\prime} \mu_{e f f}^{\prime}-\varepsilon_{\text {eff }}^{\prime \prime} \mu_{\text {eff }}^{\prime \prime}+4 \pi\left(\chi_{R}^{\prime} \mu_{e f f}^{\prime}+\chi_{R}^{\prime \prime} \mu_{e f f}^{\prime \prime}\right)|E(\omega)|^{2}$ and $\tilde{\varepsilon}^{\prime \prime}=\left(\varepsilon_{\text {eff }}^{\prime}+4 \pi \chi_{R}^{\prime}|E(\omega)|^{2}\right) \mu_{\text {eff }}^{\prime \prime}+\left(\varepsilon_{\text {eff }}^{\prime \prime}-4 \pi \chi_{R}^{\prime \prime}|E(\omega)|^{2}\right) \mu_{\text {eff }}^{\prime}$. In an LHM, $\varepsilon_{\text {eff }}^{\prime}<0, \varepsilon_{\text {eff }}^{\prime \prime}>0, \mu_{\text {eff }}^{\prime}<0$, and $\mu_{\text {eff }}^{\prime \prime}>0$,

The Stokes wave propagating along $z$ in the LHM is given by $\vec{E}\left(\omega_{S}, z\right)=\vec{A} e^{-i k_{S} z-i \omega_{S} t} \quad$ for $z>0$

with $k_{S}=k_{S}^{\prime}+i \kappa_{S}=\frac{\omega}{c} \sqrt{\tilde{\varepsilon}}$. Assuming the imaginary part of all complex quantities small compared to the real part in magnitude, we have $k_{S}^{\prime}=\left(\omega_{S} / c\right) \sqrt{\tilde{\varepsilon}^{\prime}}$ and $\kappa_{S}=\left(\omega_{S} / 2 c\right) \tilde{\varepsilon}^{\prime \prime} / \sqrt{\tilde{\varepsilon}^{\prime}}$. If the $\chi_{R}^{\prime \prime}$ term dominates in $\tilde{\varepsilon}^{\prime \prime}$, then $\kappa_{S}>0$ because $\tilde{\varepsilon}^{\prime}>0$ and $\tilde{\varepsilon}^{\prime \prime}>0$, and the Stokes wave should experience an exponential gain in the $+z$ direction although the Stokes wave vector is in the opposite direction. This is what one would expect physically. In a gain medium, the wave must grow in the direction of energy flow.

The same treatment described above should apply to other stimulated light scattering processes in nonlinear LHM.

\section{Ultra-short pulse propagation in LHM.}

Ultra-short pulses are currently available in a wide range of frequencies from $\mathrm{THz}$ to far UV. They can provide clear manifestation of the characteristic linear wave propagation effects in LHM: negative group velocity and negative refraction. We discuss qualitatively these effects. In the formal description, we can decompose the ultra-short pulse into Fourier components, follow the propagation of each component, and then sum over the components after the propagation. If the entire spectral bandwidth of the pulse sees the medium as an LHM, then the pulse will physically move in a direction opposite to the wave propagation, clearly demonstrating the negative group velocity phenomenon. If the spectral width of the pulse is broader than the bandwidth in which the LHM character of the medium prevails, the pulse is likely to split into three parts when incident into the LHM through an RHM/LHM interface. The central part of the pulse spectrum seeing the LHM will experience negative refraction at the interface, but the frequency components at the two sides of the pulse spectrum seeing no LHM will experience positive refraction. The spectroscopic study of "negatively" refracted part of pulse can be used for determination of the frequency interval within which the medium is an NRM. Interesting effects can also be expected for harmonics generation and wave mixing by ultra-short pulses: the harmonics also will propagate unusual way. As part of the spectra components of input or output experiences the LHM, the output pulses in transmission or reflection can be drastically different, in terms of energy, pulse shape, spectral composition, and direction, from those expected from an ordinary nonlinear medium. The details are complicated, depending on the spectral contents of the ultra-short pulse and the LHM. 


\section{Conclusions.}

We have proposed to use the E, D, B approach to describe linear and nonlinear wave propagation in media. Our emphasis is on NRM (or LHM). This approach avoids the usual expansion of medium response into multipoles although it can be separated into curl and non-curl parts for convenience in dealing with boundary conditions. Compared to the usual E, D, H, B approach in which the medium response is characterized by dipole polarization and magnetization with response coefficients $\varepsilon$ and $\mu$, the E.D,B approach is more general since in the optical frequency region, magnetic dipoles as usually defined no longer have their usual physical meaning and electric-quadrupole and higher-order multipole contributions may not be negligible. There is a one-to-one correspondence in description of the results using the two approaches. Specifically, we note that with either approach, linear wave propagation in NRM is characterized by negative group velocity and negative refraction. They can be illustrated by ultra-short pulse propagation in an NRM.

Using the E, D, B approach, we can calculate the simple nonlinear optical effects such as wave mixing and stimulated light scattering in NRM following the same derivations as for regular media. However, the results are qualitatively different. For example, in harmonic generation, because of improved phase-mismatch, harmonic output in reflection can be stronger than in transmission in NRM, contrary to the situation in ordinary media. With either the fundamental input or the harmonic output experiencing negative refraction, the transmitted harmonic output beam will appear on the same side of the surface normal as the fundamental input beam as dictated by the negative Snell's law. This creates a Veselago-type lens that allows SH imaging of a point source emitting at frequency $\omega$ to its mirror point appearing at frequency $2 \omega$ through a nonlinear LHM plate. Stimulated light scattering in LHM appears more like what one would expect: The growth of the stimulated radiation is always in the direction of the group velocity of the wave.

Construction of a homogeneous LHM is still a challenge. Negative refraction has been found experimentally only in artificial meta-materials composed of split ring resonator arrays in the microwave region. Photonic band-gap materials may be suitable for observation of negative refraction and negative group velocity in the optical region, but has yet to be demonstrated experimentally. In both cases, nonlinear wave mixing is probably observable if component materials of high nonlinearity are used since the effective thickness of the medium required is small. Third harmonic generation has been observed in 1-D photonic gap materials in the direction opposite to the input pump wave, but it was the results of an optical Umklapp process [17].

\section{Aknowledgements:}

VMA thanks The University of Texas at Dallas, NanoTech Institute for hospitality and support. YRS was supported by the Director, Office of Energy Research, Office of Basic Energy Sciences, Materials Science Division of the US Department of Energy under Contract No. DE-AC03-76SF00098. 
References:

1. V. G. Veselago, Sov. Phys. Usp. 10, 509-514 (1968)

2. D. R. Smith and N. Kroll, Phys. Rev. Lett. 85, 2933 (2000).

3. R. A. Shelby, D. R. Smith, and S. Schultz, Science 292, 77 (2001).

4. J. B. Pendry, Phys. Rev. Lett. 85, 3966 (2000).

5. D.R. Smith, D.Shurig, and J.B.Pendry, Appl. Phys. Lett. 81,2713-2715 (2002)

6. C. G. Parazzoli, R.B. Greegor, K. Li, B.E.C. Koltenbah, and M. Tanielian, Phys. Rev. Lett. 90, 107401(2003)

7. A. A. Houck, J.B. Brock, and I.L. Chuang, Phys. Rev. Lett. 90, 137401 (2003)

8. H. Kosaka, T. Kawashima, A. Tomita, M. Notomi,T. Tamamura,T. Sato, and S. Kawakami, Phys. Rev. B 58, R10096 (1998).

9.M. Notomi, Phys. Rev. B 62, 10696 (2000).

10. T. Ochiai and J. Sanchez-Dehesa, Phys. Rev. B. 64, 245113 (2001).

11. C. Luo, S. G. Johnson, and J. D. Joannopoulos, J.B. Pendry, Phys. Rev. B 65, 201104 (2002).

12. S. Foteinopoulou, E. N. Economou, and C.M.Soukoulis, Phys.Rev.Lett. 90, 107402 (2003)

13. L.D.Landau, E.L. Lifshits, "Electrodynamics of Continuous Media”, (Pergamon Press, New York, 1960; $2^{\text {nd }}$ edition, 1984), Chapter IX.

14. See, for example, Y.R. Shen, “The Principles of Nonlinear Optics”,(John Wiley \& Sons, New York, 1984)

15. D.A. Kirzhnitz in “The Dielectric Function of Condensed Systems”, ed. By L. Keldysh, D. Kirzhnitz, A. Dolgov, (North-Holland, Amsterdam,1989) · p.41

16.V.M. Agranovich, V.L. Ginzburg, "Crystal Optics with Spatial Dispersion , and Excitons”, (Springer, Berlin, 1984).

17. J. W. Shelton and Y. R. Shen, Phys. Rev. A 5, 1867 (1972). 